\title{
À la recherche de l'île perdue: Le Clézio à Maurice
}

\section{Claude Cavallero}

\section{Q OpenEdition}

\section{Journals}

\section{Édition électronique}

URL : http://journals.openedition.org/studifrancesi/1037

DOI : 10.4000/studifrancesi. 1037

ISSN : 2427-5856

\section{Éditeur}

Rosenberg \& Sellier

\section{Édition imprimée}

Date de publication : 1 novembre 2014

Pagination : 509-518

ISSN : 0039-2944

\section{Référence électronique}

Claude Cavallero, «À la recherche de l'île perdue: Le Clézio à Maurice», Studi Francesi [En ligne], 174 (LVIII | III) | 2014, mis en ligne le 01 novembre 2014, consulté le 18 septembre 2020. URL : http:// journals.openedition.org/studifrancesi/1037 ; DOI : https://doi.org/10.4000/studifrancesi.1037

\section{(c) $(1)(9)$}

Studi Francesi è distribuita con Licenza Creative Commons Attribuzione - Non commerciale - Non opere derivate 4.0 Internazionale. 


\title{
À la recherche de l'île perdue: Le Clézio à Maurice
}

\begin{abstract}
The evocations of Mauritius in Le Clezio's work are often part of a quest of identity that refers the reader to the author's family history. Beyond the referential framework, those biographical fictions, in particular the novel Le Chercheur d'Or (1985), develop a representation of the island that is deeply indebted to the psycho-emotional crucible of the author's childhood. Does Mauritius as it appears in the novel respond to such an intention? Does the narration in its deceptive configuration only display the patterns of a primordial imaginary? This is issue that will be worked out by the present paper. Beyond the common semantic theories and according to Jean Burgos' Poétique de l'Imaginaire, this study will consider the imaginary as a creative dynamic whose internal logic structures the mimetic immersion process involved in the advent of the fiction.
\end{abstract}

Dans le chapitre «Îles» de Raga, approche du continent invisible (2006), Le Clézio formule une question qui n'a d'anodine que la simplicité rhétorique de son énoncé: «Suis-je moi aussi d'une île?» s'interroge l'écrivain, «Ou ai-je voulu le croire?»'. Aux lignes suivantes, l'auteur évoque un rêve récurrent de son enfance dans lequel il se voyait débarquer sur une île paradisiaque où le corps des filles était éblouissant et les enfants toujours souriants, avant de conclure: «Un rêve, du banal en somme». Si l'on admet la familiarité de cette vision onirique - en dépit de l'itération qui semble motiver son surgissement après des décennies -, l'interrogation initiale demeure, et le lecteur aura du mal à lui attribuer une valeur anecdotique. Il n'échappe pas qu'une telle question - «Suis-je moi aussi d'une île?» - témoigne de l'incessante quête identitaire poursuivie par Le Clézio de livre en livre, et ainsi donc d'île en île. Cette focalisation sur l'origine nous renvoie sans détour au cadre référentiel de l'île Maurice, lequel imprègne le matériau fictionnel de plusieurs ouvrages, à commencer par le roman Le Chercheur d'or $(1985)^{2}$. Les textes de ce corpus mauricien se distinguent par leur teneur biographique attestée: ils évoquent sans ambiguïté l'histoire de la famille Le Clézio 3 . Pour les écrire, l'écrivain confie avoir puisé dans les témoignages du cercle familial autant que s'être appuyé sur ses propres séjours sur l'île. Ainsi Le Chercheur d'or fut-il rédigé à la suite de repérages effectués in situ au côté de membres de sa famille. Mais par-delà le questionnement identitaire, ces récits ne font pas que remuer et transposer une matière mémorielle. Ils ne font pas non plus qu'élaborer des représentations conformes au principe de réalité, quitte à donner aux descriptions une couleur exotique. À l'instar de nombreux romans contemporains, ces fictions biographiques mettent en question le statut de la narration, elles interrogent le rapport

(1) J.-M.G. LE ClÉZIO, Raga, approche du continent invisible, Paris, Seuil, 2006, p. 118.

(2) J.-M.G. LE CLÉZIO, Le Chercheur d'or, Paris, Gallimard, 1985; La Quarantaine, Paris, Gallimard, 1994; Révolutions, Paris, Gallimard, 2003; Ritournelle de la faim, Paris, Gallimard, 2008. L'île Maurice est par ailleurs présente dans les recueils de nouvelles Printemps et autres saisons, Paris, Gal- limard, 1989, et Histoire du pied et autres fantaisies, Paris, Gallimard, 2011.

(3) Voir la biographie de G. DE Cortanze, J.-M.G. Le Clézio, le nomade immobile, Paris, éditions du Chêne - Hachette livres, 1999. Dans la suite de la présente étude, je me référerai à l'édition en collection Folio, Paris, Gallimard, 2002. 
du fictif au factuel en brouillant délibérément les lignes de partage... Qu'advient-il, dès lors, des puissances de l'imaginaire? En quoi l'île romanesque porte-t-elle malgré tout - ou avant tout - le poinçon des schèmes d'un imaginaire singulier? Telles sont les questions auxquelles je m'attèlerai en centrant mon propos sur Le Chercheur d'or, roman où s'exprime pleinement l'impact psycho-affectif de l'enfance dans la mise en scène imaginative de l'île Maurice. Suivant cet objectif, je porterai l'examen au-delà de l'habituelle sémantique des significations afin d'envisager les manifestations induites d'une «logique de l'imaginaire» ${ }^{4}$ structurant le «processus d'immersion mimétique» ${ }^{5}$ qui préside à l'élaboration de la fiction.

Peut-être convient-il de rappeler qu'au milieu des années quatre-vingt, la publication du Chercheur d'or marque, après Désert (1980), une inflexion notable dans la création leclézienne. Cet ouvrage, renouant sans ambages avec certain plaisir lyrique de la narration, ouvre d'un coup la voie d'une écriture de la «filiation» ${ }^{6}$, un peu comme si l'entrée en scène romanesque de Maurice venait épancher le désir trop longtemps contenu d'une investigation de l'ascendance devenue soudain nécessaire. Il est aujourd'hui loisible d'observer que cette orientation rétrospective - alors inattendue sous la plume de Le Clézio - s'inscrit dans le sillage d'un épuisement des avant-gardes concomitant à l'avènement d'un nouveau «régime d'historicité» du roman, dont Dominique Viart situe précisément l'émergence au milieu de la décennie ${ }^{7}$.

Signalons que l'écriture du Chercheur d'or a pu séduire - ou au contraire éconduire - des lecteurs par le choix esthétique qu'elle oblitère au regard de l'exotisme de nombreuses descriptions - qu'il ne s'agit pas de minimiser (l'eau pure des lagons, le murmure du vent à travers les aiguilles des filaos, la magnificence des couchers de soleils...). Cet exotisme lexical appelle certes un dépassement des clichés et des stéréotypes: dans le contexte des littératures post-coloniales, cette «écriture des lointains», ainsi que l'observe Jean-Marc Moura, redonne au voyage «sa puissance de déstabilisation» et traduit un intérêt inouï pour l'histoire de l'Autre ${ }^{8}$. Il n'empêche que l'isotopie de l'île tropicale, confinant au «mythe insulaire» de la complétude originelle, agit d'emblée comme un focalisateur à forte connotation symbolique et qui produit au fil des pages une nostalgie palpable affectant le narrateur/personnage lorsqu'il se remémore, aux moments-clés de son parcours, les moments heureux de son enfance sous la varangue, dans le jardin d'Eden de la maison du Boucan. Prendre acte de cette nostalgie - plutôt que la réfuter - suppose d'envisager les forces de l'imaginaire par lesquelles s'ajoute aux significations littérales une pluralité de valences conférant au texte sa dimension poétique - voire poéthique. Ce n'est pas à travers l'enchaînement causal des événements narrés ou dans la seule matérialité des référents désignés, fussent-ils de niveau archétypal ou mythique, que peut se donner à lire «l'élaboration du surcroît de réalité» qui définit selon Jean Burgos la littérarité du texte, ce par quoi le langage «cesse de dire quelque chose pour se dire lui-même et donner réalité à l'indicible»?.

(4) J. Burgos, Pour une poétique de l'imaginaire, Paris, Seuil, 1982, p. 132.

(5) Je renvoie pour cette notion à l'ouvrage de Jean-Marie Schaeffer, qui met en lumière le rôle joué par la compétence fictionnelle dans l'économie des représentations mentales. Cf. J.-M. SCHAEFFER, Pourquoi la fiction?, Paris, Seuil, 1999.

(6) Cf. D. VIART, Récits de filiation, in D. VIaRT et B. VERCIER, La littérature française au présent, Paris, Bordas, 2005, pp. 76-98.

(7) A noter que Viart, esquissant une mise en perspective de la période pour la revue «ELFe
XX-XXI», néglige de citer les romans de Le Clézio, pourtant très significatifs de l'évolution qu'il s'emploie à circonscrire. Cf. D. VIART, Historicité de la littérature: la fin d'un siècle littéraire, in Quand finit le XX siècle, «ELFe XX-XXI», Paris, Classiques Garnier, n. 2, 2012, pp. 93-126.

(8) J.-M. MourA, Voyage, exotisme, lettres francophones et fin du XX siècle, in Quand finit le XX siècle, «ELFe XX-XXI», Paris, Classiques Garnier, n. 2, 2012, p. 174.

(9) J. Burgos, Pour une poétique de l'imaginaire cit., p. 19. 
À cet égard, le dispositif fictionnel de ce roman forme un cas d'espèce dans la mesure où, en raison peut-être de la facture déceptive de l'histoire d'Alexis et du discours d'égalité sociale qui sous-tend le récit, l'approche socio-historique semble ne pouvoir se départir de l'élan d'empathie que provoque en nous les idées humanistes de l'auteur, quitte à laisser dans l'ombre les intimations d'un imaginaire qui modalise le champ profond de l'œuvre. La quête d'Alexis se solde d'un échec auquel le déchiffrage des constellations stellaires, tout comme l'allusion répétée au mythe des Argonautes, apporte une sorte d'amplification cosmogonique. Cette amplification ne fait pourtant qu'accroître l'illusion: le chercheur d'or découvre après trente ans qu'il n'y a de trésor qu'au fond de soi, dans l'amour de la vie et la beauté du monde ${ }^{10}$. Le bilan événementiel du roman se révèle en somme très mince: le héros ne sera parvenu ni à découvrir le trésor du Corsaire inconnu, ni à refonder le domaine familial, ni même à retenir auprès de lui Ouma, la jeune «manaf» élue de son coeur...

Cet aspect dysphorique porte le lecteur à s'interroger plus avant sur un récit qui ne satisfait vraiment ni les critères de la relation de voyage, ni ceux du roman d'aventures, de la fresque historique ou du roman à thèse. S'attachant à cerner «l'herméneutique événementiale» du roman, d'après Claude Romano, Madeleine Frédéric souligne un point capital: contrairement au modèle stevensonien auquel on est tenté de l'assimiler, ce qui importe dans ce texte, écrit-elle, «c'est moins l'enchaînement des péripéties liées à la chasse au trésor que la succession des étapes jalonnant le parcours initiatique de l'advenant et la lente (re)construction de celui-ci qu'elles finiront par permettre» ${ }^{11}$. En d'autres termes, on peut dire que le réel «instaurateur-de-monde pour l'advenant $\gg^{12}$ s'assimile à l'événement lui-même et aux empreintes qu'il laisse dans l'esprit du personnage. Un glissement prévisible s'opère de l'objectif poursuivi (la découverte d'un hypothétique trésor) à l'agent même de la quête (par lequel advient l'événement). Lorsque ainsi l'aventure s'évide, se dématérialise, il ne reste plus que l'étoffe du rêve, le virtuel...

Les voix de l'imaginaire se manifestent en réalité dès les premières lignes du roman, délibérément placées sous le signe de la quête proustienne: pour le jeune Alexis, l'écoulement du Temps semble s'annihiler sous l'effet du grondement de la mer, dont la vision nocturne prend aussitôt une dimension intime: «La mer est à l'intérieur de ma tête, et c'est en fermant les yeux que je la vois et l'entends le mieux» ${ }^{13}$. La nuit, catégorie idoine de la remémoration où s'estompe la frontière entre réel et imaginaire, joue dans la filiation de Proust un rôle décisif au gré de l'itinéraire initiatique que dévoile le roman. Certes l'évocation nocturne ne remet-elle pas en cause chez Le Clézio l'identité narrative, non plus qu'elle ne plonge le lecteur dans les phénomènes associés au sommeil. Elle n'implique pas davantage une descente vers les profondeurs où puiser le sens de la réalité, l'essence des choses; mais elle fait néanmoins l'objet d'une scénographie répétitive dont l'observation des éléments naturels - la mer, la voûte céleste - institue le motif essentiel. Observer la mer de nuit, cela peut paraître une gageure, si ce n'est que cette nuit fictionnelle ne se réduit pas à l'obscurité - on sait d'ailleurs que Proust tire lui-même des images esthétiques de la mer lors des

(10) Selon un phénomène évoquant les compositions fractales, où les mêmes figures se reproduisent à différentes échelles, la configuration de l'Anse au Anglais se révèle refléter celle de l'univers. Alexis se rend compte alors qu'Ouma, qu'il avait d'abord jugé naïve, connaissait le secret depuis le tout début. Le Chercheur d'or cit., pp. 296-298.

(11) M. FRÉDÉRIC, L'écriture de l'événement dans "Le Chercheur d'or" de Le Clézio, in Au corps du texte, hommage à Georges Molinié, B. Denis et al. (dir.), Paris, Champion, 2010, p. 460. Notons que Le Clézio ne mentionne pas la référence à Stevenson, mais à un roman de Conrad, La Folie Almayer. Cf. C. CAVALlERO, Les marges et l'origine, entretien avec J.-M.G. Le Clézio [1993], «Europe», 957-958, janvier-février 2009, pp. 32-33.

(12) C. Romano, Lévénement et le monde, Paris, PUF, 1998, p. 69.

(13) Le Chercheur d'or cit., p. 14. 
nuits de clair de lune. Au demeurant, l'obscurité ne s'avère pas dangereuse chez Le Clézio - hormis le noir macabre de la guerre que découvre Alexis dans les collines de Thiepval ${ }^{14}$. Ainsi la nuit, que notre horloge biologique voue à l'effacement de la conscience - la «petite mort»-favorise au contraire une poursuite du rêve éveillé grâce à ce pouvoir nyctalope d'accroître l'acuité de la vision.

Le rêve d'Alexis ne naît pas d'une forme vide, mais plutôt d'un trop-plein d'images. Il se conjugue à la matière spontanée du souvenir, se mêle parfois à la vision prospective. La sortie inaugurale en pirogue forge l'idée du voyage maritime; d'emblée l'évocation du trésor se place au niveau du rêve ${ }^{15}$. Les limites de la vie, de la fiction et de la mort se dissolvent: pour Laure, les paille-en-queue sont «les esprits des marins morts en mer» ${ }^{16}$; tandis que les personnages du roman Lily the Nada portent «les noms d'être vivants que nous rencontrons» ${ }^{17}$. À défaut d'une lanterne magique et d'un Golo chevauchant dans une lande mythique, Mananava, tout comme le premier Combray, baigne dans l'imaginaire:

Le soir, sous la moustiquaire avant de dormir, je rêve que je suis dans un navire aux voiles gonflées qui avance au milieu de la mer sombre, et que je regarde les étincelles du soleil. J'écoute la respiration de Laure, lente et régulière, et je sais qu'elle aussi a les yeux ouverts. $\grave{A}$ quoi rêve-t-elle? Je pense que nous sommes tous sur un navire qui va vers le nord, vers l'île du Corsaire inconnu. Puis aussitôt, je suis transposé au fond des gorges de la Rivière Noire, du côté de Mananava, là où la forêt est sombre et impénétrable, et où l'on entend parfois les soupirs du géant Sacalavou qui s'est tué pour échapper aux Blancs des plantations. La forêt est pleine de cachettes et de poisons, elle résonne des cris des singes, et au-dessus de moi passe devant le soleil l'ombre blanche des paille-en-queue. Mananava, c'est le pays des rêves ${ }^{18}$.

La cellule familiale unie, la maison protectrice et son jardin céleste, son arbre «chalta» érigé en microcosme insensible aux blessures du temps, voilà qui définit une plénitude d'autant plus accomplie qu'on la sent très vite se fissurer pour laisser place à l'exil inexorable - l'installation contrainte à Forest Side. C'est bien de ces images du paradis perdu, récurrentes à longueur de texte, que procède le désir de refondation, selon un enchaînement logique des pulsions de l'imaginaire. Images interactives, tournées vers un devenir textuel, et qu'il ne s'agit pas de figer, d'isoler en archétypes si l'on veut saisir leur cohérence. «L'imagination, énonce Gilbert Durand, est dynamisme organisateur, et ce dynamisme organisateur est facteur d'homogénéité dans la représentation ${ }^{19}$.

Après la destruction de la maison du Boucan par un cyclone et l'anéantissement du projet paternel d'électrification de l'île, la famille L'Étang se voit forcée de vivre à l'intérieur des terres dans des conditions plus que rudes. Le bref chapitre consacré à l'exil de Forest Side permet d'apprécier la prégnance des représentations imaginaires au cœur de la narration. La projection dans l'image du Corsaire inconnu remplit pour Alexis une évidente fonction compulsive: «Il partageait ma vie, ma solitude. Dans l'ombre froide et pluvieuse de Forest Side, puis au Collège Royal de Curepipe, c'était avec lui que je vivais vraiment ${ }^{20}$. La vie quotidienne s'écoule dans un entier

(14) Ibid., pp. 261-262.

(15) «J'écoute mon père sans l'entendre, comme au fond d'un rêve. La légende du trésor, les recherches que l'on a faites depuis cent ans, à l'île d'Ambre, à Flic en Flac, aux Seychelles». Le Chercheur d'or cit., p. 59.

(16) Le Chercheur d'or cit., p. 64.

(17) Ibid., p. 66. L'auteur confie à Gérard de Cortanze avoir lui-même été marqué par la lecture du roman Lily the Nada, de Rider Haggard, au cours de son enfance. Cf. G. DE CorTAnZE, J.-M.G. Le Clézio, le nomade immobile [1999], Paris, Gallimard («coll. Folio»), 2002, pp. 106-107.

(18) Le Chercheur d'or cit., p. 69.

(19) G. DuRAND, Les Structures anthropologiques de l'imaginaire [1969], Paris, Dunod, 1992, p. 26.

(20) Le Chercheur d'or cit., p. 93. 
déni de réalité, dont le meilleur moment est celui du rêve que partagent le frère et la sœur d'un retour à Maurice après une vie envisagée en France: «... nous parlions du jour - lointain, évidemment - où nous reviendrions chez nous, à Maurice, comme ces aventuriers vieillis qui cherchent à retrouver leur terre d'enfance» ${ }^{21}$. Le songe éveillé, maintes fois explicité, débouche sur une rêverie énumérative des noms des navires à bord desquels le jeune homme est susceptible de s'embarquer. L'écriture de l'exil ne se projette jamais que sur le fond de son contraire, selon une syntaxe conforme à la posture du défi, de la conquête. Le narrateur se montre d'ailleurs conscient de cette dialectique imaginative lorsqu'il conclut: «Je savais pourquoi j'étais heureux chaque fois qu'un bateau hissait ses voiles et s'éloignait vers le large» ${ }^{22}$.

Il peut paraître surprenant, lorsqu'on s'arrête aux résonances autofictionnelles du texte, que le motif de l'exil surgisse d'une manière aussi abrupte au cœur de la première évocation romanesque de l'île Maurice. Voici qui donne à ce projet d'écriture longuement porté par l'auteur non l'allure d'une découverte ou d'une retrouvaille heureuse avec la terre ancestrale - d'accordailles aurait dit Glissant - mais plutôt celle, par inférence, d'un rendez-vous manqué dont on sent bien qu'aucune utopie fictionnelle ne pourrait suffire à combler l'effarement. Nous touchons-là, assurément, à l'un des schèmes les plus puissants de l'imaginaire leclézien, lequel innerve et subsume l'œuvre entière depuis ses débuts. Certes, l'exil des premiers romans s'accompagnait d'un mouvement de rejet qui tendait à le justifier: le rejet par exemple du «monde d'en bas» et de ses règles rigides pour Adam Pollo, réfugié dans Le Procès-verbal au sommet d'une colline dominant la mer ${ }^{23}$. Exil d'abord choisi pour certains personnages, comme la jeune marocaine de Désert qui cède à la tentation de l'immigration en France. Mais qu'il soit recherché ou subi, l'exil traduit toujours la perte, l'exclusion, la rupture. Il met en exergue l'appartenance préalable, originelle, matricielle, et contient toujours en germe la possibilité d'un retour, contrairement aux situations d'errance, également fréquentes chez Le Clézio, mais ne supposant ni fixitude ni possible destination ${ }^{24}$.

Le sentiment d'exil devient un principe générateur du texte. À travers la quête du trésor, l'entreprise de refondation se hisse au premier plan. En route pour l'île Rodrigues à bord de la goélette Zeta, Alexis se répète: «Je dois vaincre la destinée qui nous a chassés de notre maison ${ }^{25}$. Cette île nouvelle devient alors le métonyme de l'origine, «...comme une terre où je serais déjà allé autrefois, et que j'aurais perdue» ${ }^{26}$. $\mathrm{Au}$ fil des pages, un réseau d'images instaure ce que l'on pourrait nommer l'archipel des paradis perdus: île de Saint-Brandon pour le timonier comorien, «atoll où tout est neuf comme aux premiers jours du monde», île d'Agalega pour le capitaine Bradmer, parce qu'il s'agit de «la reine des îles [...] la plus fertile de l'océan indien» ${ }^{27}$. C'est au sens propre que l'élément maritime berce cet imaginaire de «l'éternel retour» ${ }^{28}$ selon les polarités ambivalentes attachées à sa symbolique. La mer figure l'espace protégé du lagon, la voie d'accès à «un autre monde» comparable à l'enfance ${ }^{29}$, tout comme à l'inverse les houles infernales seront responsables des naufrages où périssent les navigateurs - fussent-ils aussi aguerris que le capitaine Bradmer. Par son double mouve-

(21) Ibid., p. 100.

(22) Ibid., p. 104.

(23) Le Procès-verbal, Paris, Gallimard, 1963.

(24) Ainsi le personnage du Livre des fuites, figure même de l'errance, ne parvient-il jamais à atteindre une véritable destination au cours de son périple planétaire. J.-M.G. Le Clézio, Le Livre des fuites, Paris, Gallimard, 1969. Voir à ce propos mon étude Le syndrome de l'errance ou la fuite impos- sible, in Le Clézio, témoin du monde cit., pp. 191206.

(25) Le Chercheur d'or cit., p. 124.

(26) Ibid., p. 118.

(27) Ibid., p. 123 et p. 130.

(28) Je renvoie à l'étude fameuse de M. Eliade, Le mythe de l'éternel retour, Paris, Gallimard, 1949.

(29) Le Chercheur d'or cit., p. 134. 
ment d'avance et de ressac, la mer à la fois rapproche et distancie, ouvre les possibles et entérine l'échec ${ }^{30}$.

Au gré de cette traversée, la mer imprime son dynamisme à la phrase leclézienne - ce qui nous ramène une nouvelle fois à Proust. Mais surtout, l'objectif géographique se double d'un dessein un peu moins visible: celui de livrer une lutte qui paraît bientôt vitale contre le Temps. Les notations abondent au fil du récit de navigation pour exprimer la difficulté d'un comptage des jours, puis pour manifester le désir d'une fixation de l'instant présent. Alexis se demande: «Depuis combien de temps voyageons-nous? [...] la question se pose à moi avec une inquiétante insistance» ${ }^{31}$. Mais il souhaite au fond de lui «que cette heure ne s'achève jamais, que le navire Zeta $[\ldots]$ continue éternellement à glisser sur la mer légère ${ }^{32}$. L'effacement des repères atteint son paroxysme lorsque le capitaine lui confie la barre, comme on peut le lire à la page 125: «Je n'ai jamais connu rien de tel. Cela efface tout, efface la terre, le temps, je suis dans le pur avenir qui m'entoure». L'abolition symbolique du temps, l'impression de bascule dans un hors-temps, forme la condition même d'une reconquête pour laquelle la possession recherchée du trésor ne constitue qu'un adjuvant, un pouvoir qui précisément «ferait basculer le temps, qui abolirait le malheur et la ruine, la mort $[\mathrm{du}]$ père dans la maison ruinée de Forest Side» ${ }^{33}$. L'imaginaire temporel déployé au cours de la quête semble ainsi illustrer point pour point les définitions proposées par Gilbert Durand et Jean Burgos: il s'agit pour le narrateur de trouver une réponse face à l'angoisse existentielle liée à une expérience négative du Temps. D'où le régime antithétique d'images qui cherchent à s'immobiliser dans le présent en contrecarrant l'expression traumatique de la perte, de la mise à distance et de la séparation. Dès l'instant où le bonheur initial se trouve menacé, où l'évocation de la maison du Boucan cède à la métaphore de l'épave, et où la perspective du lendemain produit l'impression suggestive «d'être debout devant un gouffre» ${ }^{34}$, la tentation est grande de se laisser porter par le courant pour sentir, au contact de l'eau, élément primordial, que «rien n'existe plus, rien ne passe» ${ }^{35}$. Les images maternelles travaillent dès lors à une rétention des moments heureux, les inoubliables séances de lecture de l'Écriture sainte sous la varangue, les heures écoulées à rêver dans l'antre protecteur du grenier, lieu comparé à la cale d'un navire «voguant éternellement devant la ligne des montagnes» ${ }^{36}$. À travers ces rêves dont nous connaissons la prégnance, la certitude s'impose que tout doit être tenté pour endiguer l'écoulement du Temps, lequel ne peut être que fatal. D'où l'observation du narrateur aussitôt après l'ouragan: «aujourd'hui, beaucoup de temps s'est écoulé en un seul jour» ${ }^{37}$.

Dans l'Anse aux Anglais, la quête du trésor ne fera que mettre à l'épreuve du réel l'impossible combat engagé contre les forces du Temps. Aussi le rêve devient-il sans surprise le truchement nécessaire à la mise en œuvre du challenge. Le chercheur d'or en est conscient, s'écriant après quelques semaines: «ma vie est déjà semblable à ces rêves où le désir et sa réalisation ne font qu'un $\gg^{38}$. Pour parvenir à son objectif, il doit faire face à des obstacles dignes d'un récit d'initiation mythique: solitude, rigueur désertique du climat, difficultés d'orientation et de repérage, phases d'exaltation, de découragement et de délire. Hospitalisé à la suite d'une insolation, Alexis envisage de jeter l'éponge, mais très vite il reprend courage: «je ne peux me résoudre à partir. Ce serait un échec terrible; la maison du Boucan, notre vie tout entière seraient per-

(30) Concernant les représentations de la mer chez Le Clézio, voir C. CAVAllero, Le pays de la mer, in Le Clézio, témoin du monde, Paris, Calliopées, 2009, pp. 207-232.

(31) Le Chercheur d'or cit., p. 129.

(32) Ibid., p. 125.
(33) Ibid., p. 154.

(34) Le Chercheur d'or cit., p. 14.

(35) Ibid. p. 18.

(36) Ibid., p. 33.

(37) Ibid., p. 88 .

(38) Ibid., p. 172. 
dues pour Laure et pour moi» ${ }^{39}$. Il comprend dès lors que ce qu'il est venu chercher à Rodrigues dépasse ses propres limites: «c'est une force plus grande que la mienne, un souvenir qui a commencé avant ma naissance» ${ }^{40}$. À l'évidence, nous touchons-là le point nodal par lequel la fiction s'embraye sur l'expérience biographique dévoilée dans Voyage à Rodrigues (1986) ${ }^{41}$. Parvenu sur les lieux du trésor introuvable, Le Clézio explique:

Il y a quelque chose de dur dans ce pays, dur et hermétique. Je ne peux m'empêcher de penser à l'échec de mon grand-père. Ce trésor qu'il a cherché ici pendant plus de trente ans, et qui a occupé ses pensées jusqu'à sa mort, ce trésor dans lequel il avait placé tous ses espoirs, tous ses rêves, qui devait lui permettre de racheter la demeure familiale et rembourser ses dettes, ce mirage, cette chimère lui ont échappé, se sont refusés à lui. L'or est resté hors d'atteinte, à la fois proche et inaccessible, pareil à un éclat de lune, ou au reflet d'un objet perdu au fond d'un lac ${ }^{42}$.

Ces lignes permettent d'apprécier l'enjeu de l'immersion fictionnelle du point de vue de la mythologie familiale. La quête sans issue menée par le grand-père est celle d'un «homme qui avait perdu tous ses biens, dépouillé par ses proches, chassé de sa maison natale» ${ }^{43}$. L'on sait que ce déboire successoral mal élucidé et resté ancré dans les mémoires. Il fut même à l'origine de la diaspora d'une partie de la famille Le Clézio à travers le monde ${ }^{44}$. L'enquête biographique atteste le poids des références à Maurice dans le creuset éducatif des jeunes années de l'auteur: un sentiment d'étrangeté, d'inappartenance, voire de bannissement a imprégné dès la petite enfance l'imaginaire du futur écrivain ${ }^{45}$. Dans l'appartement niçois, Maurice faisait partie du quotidien, à travers l'évocation de la maison Euréka ${ }^{46}$, à travers les histoires rituelles contées par la grand-mère, à travers ces lettres écrites par de lointaines cousines, à travers des objets aussi, comme les aquarelles du fameux grand-père. Gérard de Cortanze montre à quel point cette «mémoire de l'origine» hanta l'enfance de l'écrivain, à quel point elle façonna l'habitus de la famille lorsque le père revint d'Afrique en 1952. Etre élevé comme un jeune mauricien dans la ville de Nice en plein vingtième siècle explique la différence, le sentiment de marginalité alors ressentis par l'adolescent et dont il gardera l'empreinte psychologique.

Ce poids du contexte doit être pris en compte si l'on veut appréhender l'émergence de l'imaginaire au sein du texte. Mais l'on aurait tort de percevoir ici une influence directe, comme mécanique, incitant à une lecture purement mimétique. Ce serait méconnaître le rôle structurant de l'activité imaginative en ce qu'elle mêle aux intimations émanant du milieu social les pulsions propres de l'individu selon une procédure dont les modalités restent difficiles à appréhender pour l'écrivain luimême. Ne s'exprimant que fort peu sur l'imaginaire en tant que tel, Le Clézio note

(39) Ibid., p. 185.

(40) Ibid., p. 186.

(41) J.-M.G. LE ClÉzIo, Voyage à Rodrigues, Paris, Gallimard, 1986.

(42) Voyage à Rodrigues cit., p. 22.

(43) Voyage à Rodrigues cit., p. 23.

(44) L'auteur précise dans son journal: «Tous les enfants de Sir Eugène quittent le domaine où ils sont nés, où ils ont grandi heureux. Les garçons voyagent, vont au bout du monde, en Amérique, en Afrique, en Europe. Les filles sont vouées à la pauvreté». Cf. Voyage à Rodrigues cit., p. 113.

(45) L'écrivain explique à Gérard de Cortanze: «depuis ma naissance, j'ai été élevé avec cette idée, que ma famille et moi n'étions ici, en France, que provisoirement et que, même si nous restions à Nice, nous repartirions un jour pour Maurice». G. DE Cortanze, J.-M.G. Le Clézio, le nomade immobile cit., pp. 271-272.

(46) Dans Voyage à Rodrigues, Le Clézio consacre plusieurs pages à l'évocation de cette maison mythique, qu'il décrit comme «le lieu le plus important de [sa] famille». Voyage à Rodrigues cit., pp. 117-121 (p. 117 pour la citation). La maison référentielle a par ailleurs inspiré à l'écrivain la Rozilis décrite par la tante Catherine dans Révolutions. Cf. Révolutions cit., pp. 47, 114-115. 
simplement: «lorsque j'écris, j'ai le sentiment que je suis en présence d'une invasion d'imaginaire» ${ }^{47}$. L'imagination s'assimile selon lui à la mémoire. Ainsi confie-t-il à son biographe: «J'ai eu souvent l'impression d'inventer, mais je pense qu'en fait, lorsqu'on écrit, on n'invente pas. On est toujours propulsé par une mémoire qui appartient quelquefois aux autres, à ce que les autres vous ont raconté, à ce que vous avez entendu, mais il s'agit en fin de compte toujours de mémoire: une poussée assez involontaire ${ }^{48}$. Si cet épitexte s'avère utile, son éclairage peut aussi éconduire le critique, car en mettant le zoom sur l'expression des seules réminiscences, il nous éloigne une nouvelle fois d'une approche dynamique de l'imaginaire, occultant ces «conflits générateurs de forme» que s'emploie à décrire Jean Burgos ${ }^{49}$. Quelle que soit la spécificité du substrat mauricien dans l'œuvre de Le Clézio, le risque est patent de renouer ici avec la plus insipide des lectures biographiques: l'œuvre-reflet de la vie de l'auteur. Pour autant que les représentations de Maurice et de la trajectoire familiale dont l'écrivain a été bercé jouent un rôle indéniable dans la projection qu'élabore la diégèse, de même que l'expérience perceptive dont l'imagination porte la marque résiduelle, il serait réducteur de confiner la créativité romanesque à ces seuls éléments, et de ne lire en somme le texte qu'à la lueur de son contexte, comme le fait notamment Michèle Gazier en s'attachant à cerner la «vérité de Maurice» sous le calque des cartes postales et des «fictions de la mémoire» ${ }^{50}$.

Bien au-delà de la révélation documentaire réduisant l'œuvre à sa composante mimétique, l'imaginaire, dimension essentielle du texte de fiction, enrichit l'écriture de significations nouvelles. Qu'on lui attribue un fondement organique, comme le fait Burgos, ou plutôt pratique, comme le fait Descola, le schème, opérateur de convergence, apparaît pour ainsi dire le logiciel système de ce processus ${ }^{51}$. C'est ainsi que le stress et l'insécurité liés à la situation d'exil, psychiquement affrontés par l'auteur depuis la prime enfance - pour ne pas spéculer sur un souvenir antérieur à la naissance qu'évoque le narrateur ${ }^{52}$ - ont pu déterminer l'équivalent d'une «attitude» au sens psychosocial, à savoir une propension à produire des images similaires en fonction d'une situation donnée. Toutefois ces images n'ont pas vocation à reproduire le stress, on le voit par l'omniprésence des notations sensorielles, par le surgissement de la beauté du corps dans les évocations. L'image poétique, dans sa fraîcheur, aspire aux commencements: l'attrait pour les lieux de partance (estuaires, quais, embarcadères...), participe ainsi dans Le Chercheur d'or d'une imagerie récurrente qui accorde le déracinement au besoin d'évasion, signe d'un mal-être identitaire. Dès

(47) J.-M.G. Le Clézio, le nomade immobile cit., p. 292.

(48) Ibid., p. 73.

(49) Pour une poétique de l'imaginaire cit., p. 15.

(50) M. Gazier, Paysages de Maurice, in Le Clézio, passeur des arts et des cultures, T. Léger, I. Roussel-Gillet et M. Salles (dir.), Rennes, PUR, 2010, p. 26.

(51) Dans sa définition du schème de l'imaginaire, Jean Burgos intègre la notion kantienne de «représentation intermédiaire» jouant un rôle de jonction entre l'intelligible et le sensible, de même que l'approche du psychologue Burloud, pour qui le schème dénote «une tendance à articulations multiples $[\ldots]$ une forme qui s'inscrit progressivement dans une matière et qui, en s'y inscrivant, l'organise». J. BuRGOS, Les schèmes et leurs fondements, in Pour une poétique de l'imaginaire cit., pp. 116-122; Kant, Analytique transcendantale, in Critique de la raison pure, livre II, chap. 1, Du schématisme des concepts purs de l'entendement; A.
Burloud, Psychologie, Paris, Hachette, 1948, p. 27. Entre l'approche structurale (Durand) et poétique (Burgos), Descola propose la voie médiane de «schèmes pratiques intériorisés, synthétisant les propriétés objectives de toute relation possible avec les humains et les non-humains». Cf. P. Descola, Par-delà nature et culture, Paris, Gallimard, 2005, p. 139.

(52) Cette mémoire prénatale est évoquée de manière explicite à la page $186 \mathrm{du}$ roman. L'auteur y fait également allusion dans son journal. Cf. Voyage à Rodrigues, J.-M.G. Le Clézio cit., p. 134. On ne peut s'empêcher de penser ici à ce «point obscur» qu'évoque l'écrivain dans un entretien, «un point que je ne peux pas voir, mais que je sens au fond de moi, et qui me conduit jusqu'au XIX ${ }^{\mathrm{e}}$ siècle, à l'époque de l'esclavage, au temps où l'Occident affirmait en toute quiétude sa domination sur le reste du monde». C. CAVALLERO, «Les marges et l'origine», entretien avec J.-M.G. Le Clézio [1993], «Europe», 957-958, janvier-février 2009, p. 38. 
le début, Alexis ne peut se contenter d'admirer la ligne des brisants qui bornent le lagon; selon une modalisation descriptive très significative, il lui «faut» toujours imaginer l'escapade en pirogue. L'insertion d'items qui semblent ne remplir d'abord aucune fonction dans l'économie du récit (le vol apaisant des pailles-en-queue, les pyramides de pierre le long des champs, la figure légendaire de Sacalavou), s'organise selon un algorithme où le schème de l'exil motive des images alternatives d'aliénation, de révolte et de liberté. Les relations entre personnages épousent cette syntaxe fonctionnelle: la sollicitude spontanée d'Alexis envers Denis, petit-fils du cuisinier et descendant d'esclaves malgaches, s'affirme d'emblée, de même que sa compassion appuyée pour ces travailleurs agricoles qu'il nomme «les martyrs de la canne» ${ }^{53}$. À l'inverse de son cousin Ferdinand, dépositaire légitime des attributs du pouvoir colonial, Alexis est attiré par le petit peuple des exilés, des clandestins, ainsi les manafs qu'il a l'opportunité d'approcher à travers Ouma à Rodrigues. Nous voici conduits à nous demander, à la suite de Jean-Philippe Pierron, si «l'augmentation iconique du monde par l'expérience poétique» du rêve de reconquête ne contribue pas à féconder une «responsabilité éthique» de l'écriture dont on sait qu'elle s'épanouit au fil de l'œuvre de Le Clézio ${ }^{54}$.

La mise en fiction de Maurice est loin d'inspirer dans Le Chercheur d'or la sérénité d'un possible retour à l'origine. Maurice reste l'île perdue de Le Clézio, tel un trésor symbolique qui n'aura jamais livré son énigme. Mais la mise à l'épreuve des forces combatives de l'imaginaire a permis au protagoniste d'augmenter l'intensité de sa présence au monde en améliorant la compréhension de sa relation à ses proches, et surtout à l'Autre - dont Ouma se veut l'incarnation exemplaire. Le motif de la lutte - illustré dans le rêve de trésor sous une forme chimérique, mais aussi montré dans son extrémité la plus absurde: la guerre - permet l'infléchissement de la posture héroïque initiale de révolte contre le Temps pour s'ouvrir à une modalité de progrès: «L'infinitude est ici cherchée non plus dans un temps figé en éternel présent, ni dans un refuge hors du temps, mais dans l'œuvre même du temps dont la circularité est délibérément perçue comme créatrice et dont la vectorialité prend elle-même un sens» ${ }^{55}$. Ce temps «réalisé» qu'évoque Le Clézio dans Voyage à Rodrigues ${ }^{56}$ n'est pas très éloigné du temps «retrouvé» par le narrateur proustien, tout comme, on l'aura compris, le schème de l'exil suggère en filigrane celui de l'abandon...

Scellant la boucle herméneutique du récit, le progrès psychique accompli par Alexis doit être envisagé dans sa conformité au climat axiologique global du roman, et en particulier dans le rapport qui s'insinue entre les valeurs éthiques et les schèmes de l'imaginaire leclézien. Ces valeurs, qu'évoque Jean-Philippe Pierron (à propos de la préface à l'édition française du livre d'Aldo Léopold rédigée par Le Clézio en $1995)^{57}$, semblent en effet témoigner du pouvoir «émergeant» de l'imaginaire comme «structure d'accueil disponible à l'être-là du monde, précédant et nourrissant la volonté responsable» ${ }^{58}$. En ce sens, il importe de souligner que l'attitude compatissante du chercheur d'or à l'égard des défavorisés de toute origine ethnique ne relève ni du dogme religieux, ni d'une réflexion philosophique ou d'une ligne politique susceptible de prendre pied dans les marges du récit. C'est en vain que l'on chercherait un discours additif à la narration et par lequel l'écrivain s'emploierait à la promo-

(53) Le Chercheur d'or cit., p. 61.

(54) J.-P. Pierron, Les puissances de l'imagination, Paris, Cerf, 2012, p. 66. Concernant «l'engagement éthique» de Le Clézio, voir C. CAVALLERo, J.-M.G. Le Clézio et la question de l'engagement, Horizons lecléziens, actes du colloque de Grenade, Toulouse, Interlignes, 2009, pp. 195-216.

(55) Pour une poétique de l'imaginaire cit., p. 128.
(56) Voyage à Rodrigues cit., p. 130.

(57) A. LEOPOLD, Almanach d'un conté des sables (A Sand Country Almanach and Sketches Here and There), traduction de l'américain par Anna Gibson, 1949, avec une préface de J.-M.G. Le Clézio, Paris, Aubier, 1995.

(58) Les puissances de l'imagination cit., p. 86. 
tion de ses «idées», comme on pourrait le croire à la lecture de certains critiques. Pour comprendre enfin la manière dont l'image poétique possède en soi une portée éthique - et donc «poéthique»-, c'est au schème fondateur de l'exil qu'il convient encore de revenir: chez Le Clézio, l'exil s'accompagne d'une hantise de la privation, une peur de manquer dont on trouve des illustrations explicites dans Ritournelle de la faim. En soulignant l'importance des mots «rigueur» et «parcimonie» dans les valeurs éducatives de la famille Le Clézio, Gérard de Cortanze met le doigt sur une angoisse de la pauvreté que reconnaît l'auteur, et l'on comprend que ce ressenti profond puisse ériger des défenses psychiques à même d'investir le plan idéologique du roman par le jeu de représentations témoignant de l'intensité imageante de ce rapport au monde intériorisé depuis l'enfance ${ }^{99}$. Par la combinatoire des processus structuraux de l'immersion fictionnelle, qui interroge ici une philosophie de l'anthropos comme sujet ainsi que le note Pierron ${ }^{60}$, les enjeux poétiques du récit s'unissent aux pulsions éthiques de l'imagination, au «noyau éthico-mythique» (Ricoeur) par lequel l'entreprise romanesque se montre en l'occurrence irréductible à un discours purement intentionnel sur des valeurs interculturelles prêtes à l'emploi, pour ouvrir une brèche interprétative d'où naît l'entier plaisir d'une lecture vivifiée.

CLAUDE CAVALLERO

(59) J.-M.G. Le Clézio, le nomade immobile cit.,

(60) Les puissances de l'imagination cit., p. 72. p. 78. 